\title{
Experimental verification of field extension for non-homothetic arrays with a pupil-plane interferometer
}

\author{
I. Montilla, S. F. Pereira*, J. J. M. Braat \\ Delft University of Technology, P.O. Box 5046, NL-2600 GA Delft, The Netherlands
}

\begin{abstract}
Wide field interferometry has become a subject of increasing interest in the recent years. New methods have been suggested in order to avoid the drawbacks of the standard wide field method (homothetic mapping) which is not applicable when the aperture is highly diluted; for this reason imaging with non-homothetic arrays is being extensively studied ${ }^{1,2}$. The field of view of a pupil plane interferometer or a densified array consists only of a few resolution elements; in order to improve these systems, we developed a new method consisting of a Michelson pupil-plane combination scheme where a wide field of view can be achieved in one shot. This technique, called "staircase mirror" approach, has been described in a previous paper ${ }^{3}$ and uses a stair-shaped mirror in the intermediate image plane of each telescope in the array, allowing for simultaneous correction of the differential delay for the on and off-axis image positions. Experimental results have been obtained recovering the fringes of off-axis stars with an angular separation of approximately 1 arcmin simultaneously, and with a contrast similar to that of the on-axis reference star. With this example, we demonstrate an increase of the field of view by a factor of five, with no need of extra observation time. An algorithm to recover the visibilities from the stars focused on the edge of the steps is described and experimental results are shown that prove that a continuous wide field of view can be acquired in one shot.
\end{abstract}

Keywords: Interferometry, wide field imaging, aperture synthesis

\section{INTRODUCTION}

Until recently, it has been thought that Fizeau-type interferometers were the only way to perform wide-field imaging, since in pupil-plane interferometers the field of view consists only of a few resolution elements ${ }^{4}$. But when the aperture is highly diluted, the applicability of a Fizeau interferometer is limited by the signal-to-noise ratio, that decreases with the number of telescopes. This problem does not occur in Michelson interferometers ${ }^{5}$. In order to use this favourable property of Michelson interferometers, we developed a new approach to the pupil recombination problem, i.e., a system that uses a Michelson pupil-plane scheme with a wide field of view to be acquired in one shot ${ }^{3,6}$. We introduced a new technique, called "staircase mirror" approach, which consists of positioning a stair-shaped mirror in an intermediate image plane for each telescope in the array. In this way, the light from stars arriving at different angles on the telescopes is imaged on different steps on the staircase mirror (see Fig. 1). Consequently, the extra path length introduced by the staircase mirror provides simultaneous correction of the Optical Path Difference $(O P D)$. The shape of the mirror depends on the baseline and the pointing direction of the telescopes, and it must be actuated during the observation as the entrance pupil geometry varies with the hour angle. Given the discontinuous nature of the staircase mirror, it may appear that the correction of the $O P D$ in such a way would yield a discontinuous field of view. When an object is focused on the edge of a step, the light is reflected from two steps simultaneously, implying a different phase shift for each part. The effect on the detected light distribution after combining the beams is that instead of having one fringe set, with one maximum at the zero path position, there are two fringe sets, each with a relative maximum. Each set has a relative contrast, and the addition of the contrast of the two components of the fringe results in the expected contrast of the source multiplied by a factor that depends on the bandwidth and the baseline; with the aid of this factor the visibility can be retrieved.

This paper is organized as follows: the experimental setup built to verify our wide-field concept is described in Section 2 , and the results obtained with it are shown in Section 3. In Section 4 we present the algorithm to retrieve the visibility of a

\footnotetext{
*s.f.pereira@tnw.tudelft.nl
} 
source that is focused in the edge of a step, and show the experimental results that prove that using this algorithm the visibility is completely recovered.

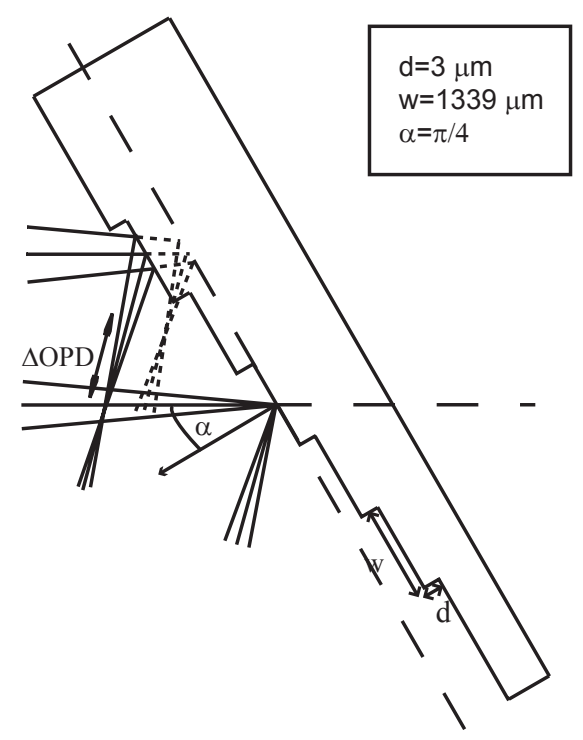

Figure 1. $O P D$ equalization; the light from different directions in the field is focused on different steps which introduces an extra $O P D$ as a function of the field angle.

\section{EXPERIMENTAL SETUP DESCRIPTION}

We have designed and implemented a tabletop setup consisting of a two-telescope Michelson-type interferometer. In the focal plane of one of the arms of the interferometer a staircase mirror was placed. A scheme of the setup has been plotted in Fig. 2 and it consists of three main blocks: the star simulator, the interferometer and the beam combiner.

The star simulator consists of a Xenon arc lamp with a set of filters that reduces its spectrum to a bandwidth of $150 \mathrm{~nm}$ (500-650 $\mathrm{nm})$, resulting in a coherence length of $2.2 \mu \mathrm{m}$. The light is imaged onto the star mask, which consists of a set of pinholes of $5 \mu \mathrm{m}$, where each pinhole defines one star. In the experiment described here the illuminated mask has four pinholes, distributed in a square with separation of $150 \mu \mathrm{m}$. The size of the holes ensures full spatial coherence for the beams over the entire bandwidth. The light from two pinholes is not coherent, ensuring two independent sources as is the

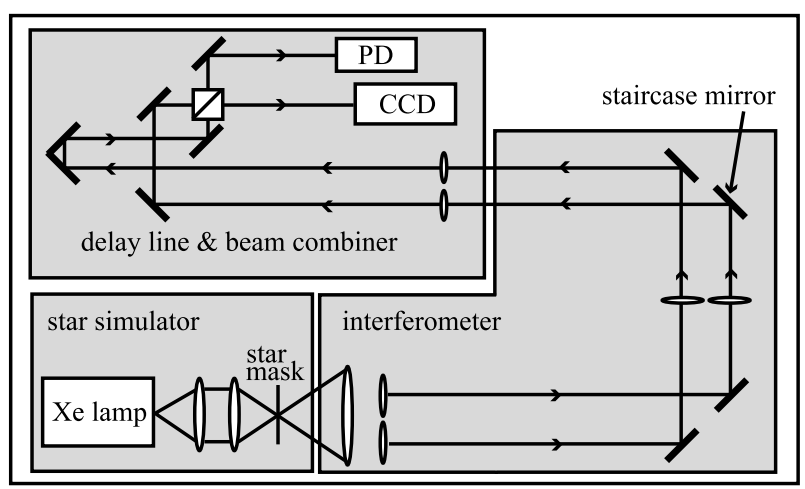

Figure 2. Scheme of the experimental setup consisting of three blocks: the star simulator with the white light source and the star mask, the interferometer, with the staircase mirror in one of the arms, and the beam combiner with the delay line. A detector (PD) was placed in one of the outputs of the beam splitter and a CCD camera at the other to control the pupil position. The delay line (DL) was connected to a piezo driver and to a signal generator to modulate the path producing temporal fringes. 
case with real stars. The light from the star mask is collimated in such a way that in the entrance pupil of the telescopes we have four beams, simulating four distant objects in the sky. Two of these objects have an external OPD equal to zero, therefore they are considered on-axis objects. The other two have an external $O P D$ different than zero, hence for the interferometer they are off-axis objects, with the off-axis angle being approximately 1 arcmin.

The two interferometer beams are obtained by wavefront division by means of two apertures of 20 mm diameter, and the separation between the apertures ("baseline") is $30 \mathrm{~mm}$. After each aperture a telescope objective is placed, and at the focal plane of one of the interferometer arms a staircase mirror is set, such as drawn in Fig. 1. The staircase mirror introduces a temporal delay to the imaged spots that depends on the incidence angle of the incoming beam at the entrance pupil of the telescope. The introduced delay depends on the tilt angle of the mirror. We have chosen a tilt of $45^{\circ}$ so that the symmetry between both arms is easily maintained. The width of the step depends on the effective focal length, $F$, of the telescope objective and was chosen so that the point spread function of the focused spot is several times smaller than the width of the step. In the actual configuration the effective focal length is $6.563 \mathrm{~m}$ and the system is free of lateral and axial chromatic aberration. The depth of the step, $d$, is related to the width, $w$, and the tilt angle, $\alpha$, by the equation:

$$
\frac{d}{w}=\frac{B}{2 F} \tan \alpha
$$

where $B$ is the baseline or separation between the telescopes. The staircase mirror was manufactured at Philips Research Laboratories (Eindhoven, The Netherlands) with a diamond turning machine (DTM) using a chisel of $3 \mu \mathrm{m}$. For the chosen step width of $1339 \pm 6 \mu \mathrm{m}$ both the on and the off- axis beams are focused close to the centre of their respective steps. The corresponding depth of the steps is $3 \pm 0.2 \mu \mathrm{m}$.

The staircase mirror holder can be rotated, so that the steps can be set either perpendicular to the baseline, in order to correct the external optical path, or parallel to it, which corresponds to an uncorrected situation (i.e., no staircase mirror).

The light is collimated again before entering the delay line and beam combiner block. Two mirrors on top of a piezo stage, set in one of the interferometer arms, provide a delay line to modulate the optical path. The beams overlap at a beam splitter/combiner cube. The two outputs of the beam splitter are detected by a low noise photo detector and a CCD camera that measures the modulated fringes and controls the pupil position, respectively.

\section{EXPERIMENTAL RESULTS}

\subsection{Assembly, alignment and calibration}

The setup was assembled starting from the star simulator, then both interferometers arms, the delay line mirrors and the beam combiner. A He-Ne laser set before the star mask was used for the alignment. The piezo stage of the delay line was actuated with a triangle function of 4 volts amplitude, equivalent to a modulation of the path of approximately $9 \mu \mathrm{m}$. The
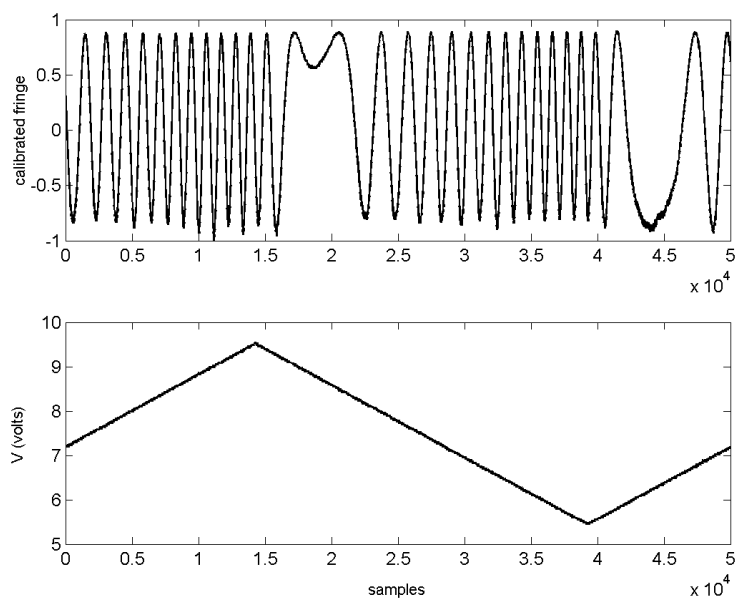

Figure 3. Experimental laser fringes, used for alignment and calibration. One period of the ramp is equivalent to a path modulation of $9 \mu \mathrm{m}$. 
fringes produced by the coherent interference of the beams were detected with the photo detector, as well as the intensity of each beam for calibration of the visibility. The maximum visibility obtained with the laser was $0.92 \pm 0.02$, as seen in the calibrated fringe pattern shown in Fig. 3. Since all the mirrors in the setup have a surface quality of $\lambda / 20$, higher contrast fringes should be expected from a monochromatic point source. The limited observed contrast could be due to two reasons: misalignment or the fact that the observed source is partially resolved by the baseline. In order to calibrate the instrument for the white light measurements, it is necessary to know if the object observed is not a point source. Direct measurements of the distance from the star mask to the collimating lens, result in a distance of $480 \mathrm{~mm}$, i.e., $20 \mathrm{~mm}$ shorter than the focal length of the lens. Consequently, the collimating lens is not relaying the star mask at infinity, but producing a virtual image of a star with $0.9 \times 10-5$ rad angular diameter. The visibility at $632 \mathrm{~nm}$, observing with a baseline of $30 \mathrm{~mm}$, is expected to be 0.95 . Given this number, the residual reduction would be attributed to alignment or surface errors. The total reduction due to these factors is about $3 \%$. For a partially resolved source, the visibility depends on the wavelength; given the measured values of the visibility with the laser radiation the expected visibility for the white light source, with a central wavelength of $575 \mathrm{~nm}$, is about 0.90 . When measuring the white light fringes, first results showed that fringes for the on-axis and off-axis stars were observed simultaneously when the dividing lines of the steps of the staircase mirror are perpendicular to the baseline (i.e. with correction of the $O P D$ ). But the contrast was 0.26 , which is a quite low value. The reason for such a low contrast as compared with the contrast measured with the laser is attributed to dispersion produced by the various optical elements. The different types of glass present in the setup are BK7, F2, SF2 and SF5. In order to correct the dispersion, two types of glass, expected to give the larger contributions to the dispersion (BK7 and F2), were inserted to the interferometer's arms. By tilting the glass plates and observing the fringes, we obtained after optimization a visibility $0.82 \pm 0.02$.

\subsection{Measurements}

After correction for the dispersion, we measured the fringes corresponding to the four stars in the star mask. In the first set of data the staircase mirror was placed with the steps parallel to the baseline, so that no correction of the external $O P D$ is introduced. The calibrated fringes are shown in Fig. 4, obtained with a total scanning distance of $9 \mu \mathrm{m}$. The fringe from the on-axis star (Fig. 4a) has a visibility of $0.82 \pm 0.02$. The peak of the fringe from the off-axis star is not observed, since it is not in the field of view of the interferometer, and the visibility was $0.14 \pm 0.02$ (Fig. $4 \mathrm{~b}$ ). Similar results are found with the other two on- and off-axis stars. In the second set, we place the mirror with the steps perpendicular to the baseline, so that correction of the external OPD does take place. The results are shown in Fig. 5. The visibility calculated from the calibrated fringe of the two on-axis stars is again $0.82 \pm 0.02$ (Fig. 5a and Fig. 5c). The visibility of the two off-axis stars is $0.74 \pm 0.02$ (Fig. 5b and Fig. 5d). This value, as compared with the visibility of the off-axis star without correction (Fig. $4 \mathrm{~b}$ ), clearly demonstrates the effect of the $O P D$ correction due to the steps. The relative phase, $\varphi$, that gives the position of
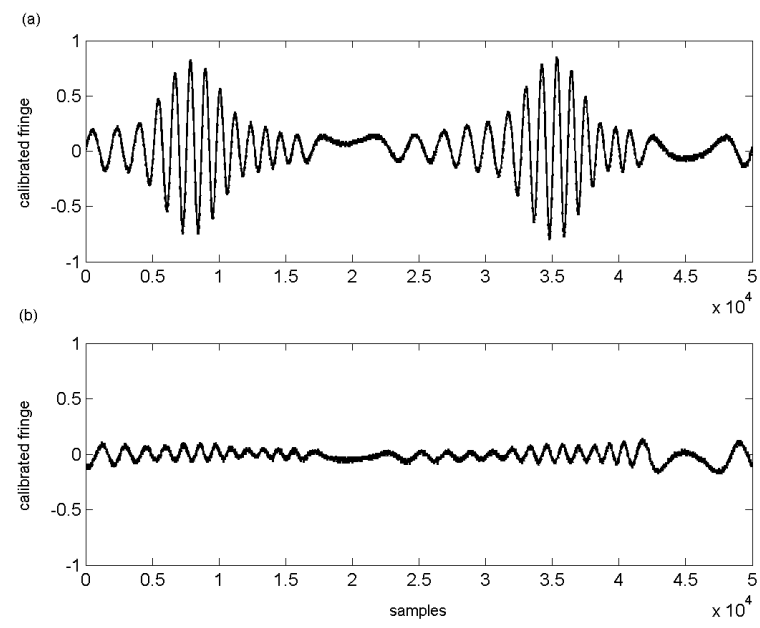

Figure 4. White light fringes when the mirror has the steps parallel to the baseline: (a) the on-axis calibrated fringe, (b) the offaxis calibrated fringe. The off-axis star fringe is out of the interferometric field of view; its peak is not observable. 
the off-axis star with respect to the on-axis one, can be calculated by measuring the distance from the peak of the off-axis fringe pattern to the peak of the on-axis pattern, $n_{\mathrm{p}-\mathrm{p}}$, and knowing in which step $n$ the off-axis star is focused:

$$
\varphi=\frac{1}{B}\left(2 n d \cos \alpha+n_{\mathbf{p}-\mathbf{p}}\right)
$$

where $n_{\mathrm{p}-\mathrm{p}}$ can be both positive or negative. The relative phase measured from the fringe pattern is $(3.3 \pm 0.1) \times 10^{-4} \mathrm{rad}$, which closely agrees with the calculation of the phase by direct measurement of the experimental parameters.

The visibility of the off-axis fringes is not equal to the visibility of the on-axis fringe pattern again because of the dispersion effects. For the data shown in Fig. 5, we set the on-axis star at the centres of the achromats and the dispersion correction was optimized for this direction. Therefore, the off-axis stars hit the achromats off-axis and the dispersion correction is not optimum in this case. By moving the stars in such a way that they hit the achromats symmetrically, we observe the same visibility, $0.80 \pm 0.02$, in both cases.
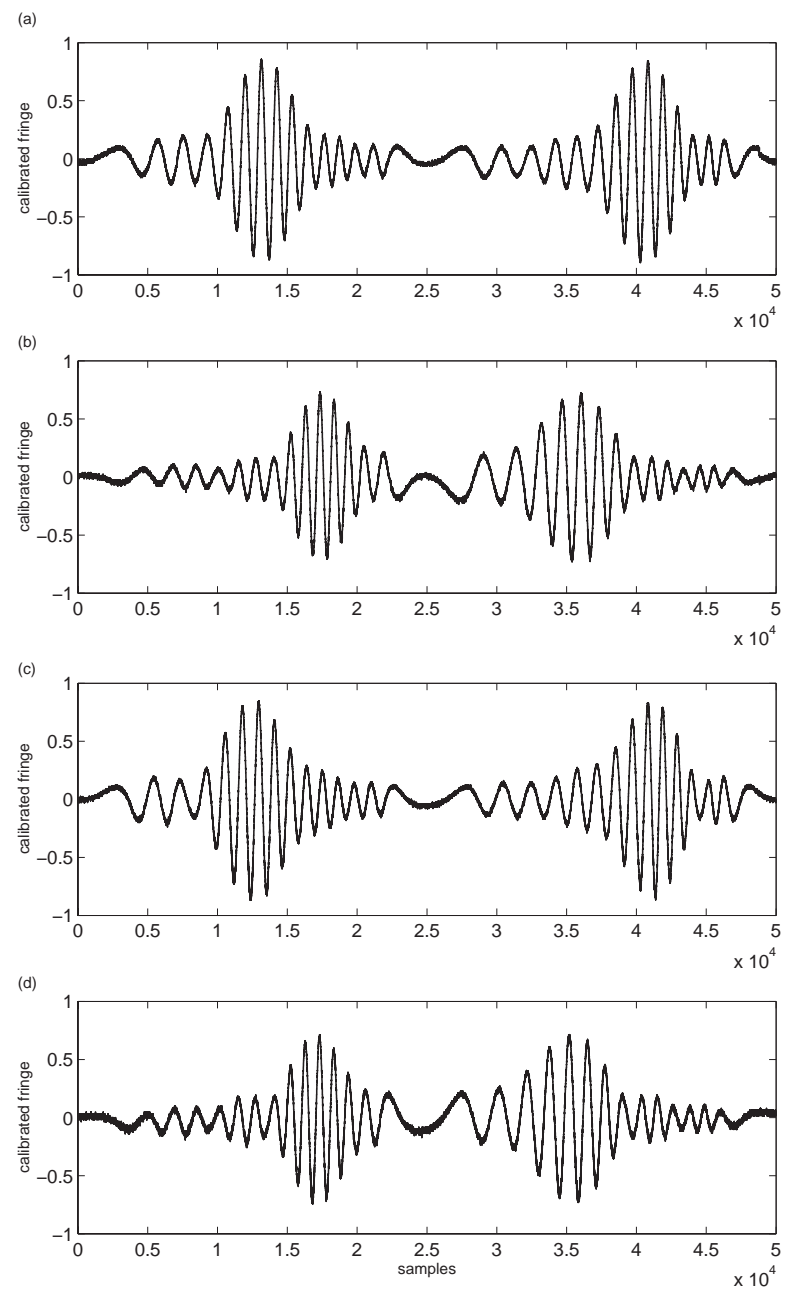

Figure 5. White light fringes when the steps of the mirror are perpendicular to the baseline: (a) and (c) calibrated fringes from the on-axis stars, (b) and (d) calibrated fringes from the off-axis stars. The interferometric field of view has been extended and the peaks of the fringes of the off-axis stars are observed. 


\section{VISIBILITY RETRIEVAL OF A SOURCE FOCUSED IN THE EDGE OF A STEP}

\subsection{Introduction}

In the case of a source focused on the edge of a step, the interference of the beams results in two sets of fringes, both with a contrast smaller than the one corresponding to the star focused in the middle of the step. In this situation, the internal phase introduced by the steps is not the same along the entire PSF. The phase difference introduced by the step is given by $\kappa_{0} d_{\text {eff }}$, where $d_{\text {eff }}=2 d \cos \alpha$ and $\kappa_{0}$ is the wave number corresponding to the central wavelength $\lambda_{0}$. The light reflected from each step will result in two relative maxima of the luminosity: the first one occurs when the phase is 0 and $-\kappa_{0} d_{\text {eff }}$ for the light focused on the first and second steps, respectively, and the second one when the phase is $\kappa_{0} d_{\text {eff }}$ and 0 for the light focused on the first and second steps, respectively. Correspondingly, there will also be two relative minima. The resulting fringe pattern is composed of two sets of fringes, each of them with visibilities $\mu_{1}(B)$ and $\mu_{2}(B)$, respectively. Adding them we obtain ${ }^{7}$ :

$$
\mu_{1}(B)+\mu_{2}(B) \cong f \mu(B),
$$

where $f$, the so-called edge factor, is given by

$$
f=\frac{f_{\mathbf{f}}+f_{-\mathbf{f}}}{2}
$$

with

$$
\begin{gathered}
f_{ \pm \mathbf{f}}=\frac{1+\mathbf{A}+\cos \left(\kappa_{0} d_{\text {eff }}\right)\left(\mathbf{E}+\mathbf{C}_{ \pm \mathbf{c}}\right)}{2} \\
\mathbf{A}=\sin \mathbf{c}\left(\frac{\pi \lambda_{0}}{L_{c}}\right) ; \quad \mathbf{C}_{ \pm \mathbf{c}}=\sin \mathbf{c}\left(\frac{\pi d_{\text {eff }}}{L_{c}} \mp \frac{\pi \lambda_{0}}{2 L_{c}}\right) ; \quad \mathbf{E}=\sin \mathbf{c}\left(\frac{\pi d_{\text {eff }}}{L_{c}}\right) .
\end{gathered}
$$

The relative phase, $\varphi$, that gives the position of the star focused in the edge with respect to a reference one, can be calculated by measuring the distance from one of the peaks of the fringe sets to the peak of the reference fringe pattern. If $n_{\mathbf{p}_{1}-\mathbf{p}_{0}}$ is the distance from the peak of the first fringe set to the reference fringe pattern, and $n_{\mathbf{p}_{2}-\mathbf{p}_{0}}$ the distance from the peak of the second fringe set to the reference fringe pattern, knowing between which steps, $\mathrm{n}$ and $\mathrm{n}+1$, the star is focused, the relative phase is

$$
\varphi=\frac{1}{B}\left(n d_{e f f}+n_{\mathbf{p}_{1}-\mathbf{p}_{0}}\right)=\frac{1}{B}\left((n+1) d_{\mathbf{e f f}}-n_{\mathbf{p}_{2}-\mathbf{p}_{0}}\right),
$$

where $n_{\mathbf{p}_{1}-\mathbf{p}_{0}}$ and $n_{\mathbf{p}_{2}-\mathbf{p}_{0}}$ can be either positive or negative but have always opposite sign.

\subsection{Experimental results with a $30 \mathrm{~mm}$ baseline}

The fringes were measured for five different positions of a star with respect to the edge of the step. First, the star was situated on-axis and at the centre of one step, obtaining one set of reference fringes. The visibility of this set of fringes was used as reference. Then, the star mask was translated in such a way that the star was placed close enough to the edge so that a second fringe set started to appear. Subsequently, the star was moved to the edge between the steps, resulting in two sets of fringes with similar contrast. As the star is moved further towards the centre of the next step, the contrast of one of the sets increases while the other one decreases in a way that the total contrast stays constant. When the star is focused close to the centre of the next step, the interference fringes form one set again.

The measured calibrated fringes, using a bandwidth from $500 \mathrm{~nm}$ to $650 \mathrm{~nm}$, are shown in Fig. 6. When the star is focused close to the centre of the step, the visibility is $0.82 \pm 0.02$, as shown in Fig. 6a. In Fig. 6b, the star is placed close to the edge and a second set of fringes begins to appear; the addition of the two visibility components is equal to 0.92 . Fig. $6 \mathrm{c}$ shows the calibrated fringe measured when the star is focused approximately at the centre of the edge, so that the two compo- 
(a)

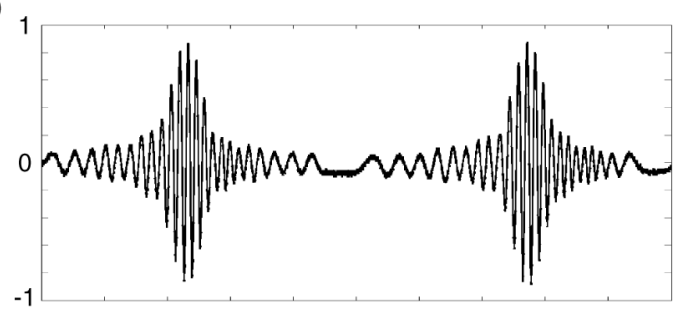

(b)

(c)
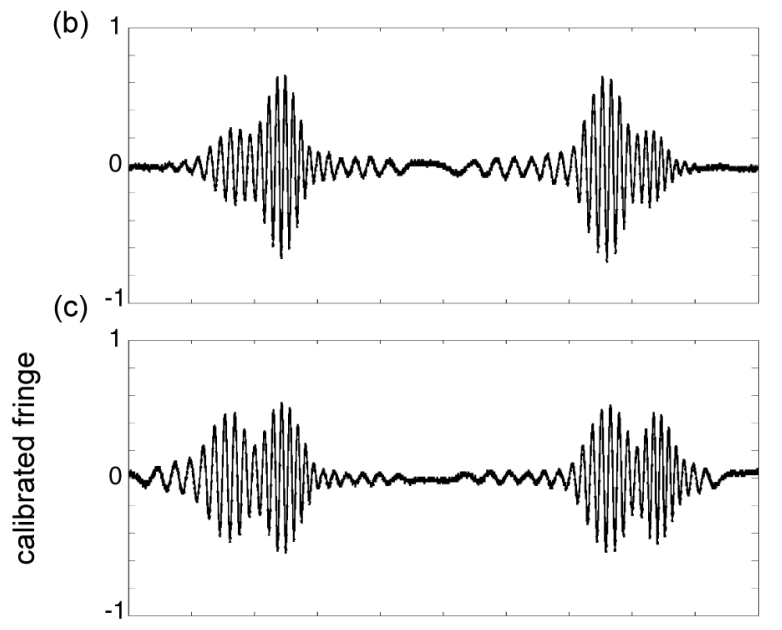

(d)

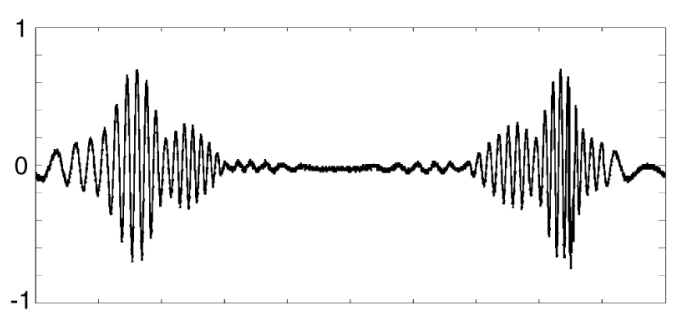

(e)

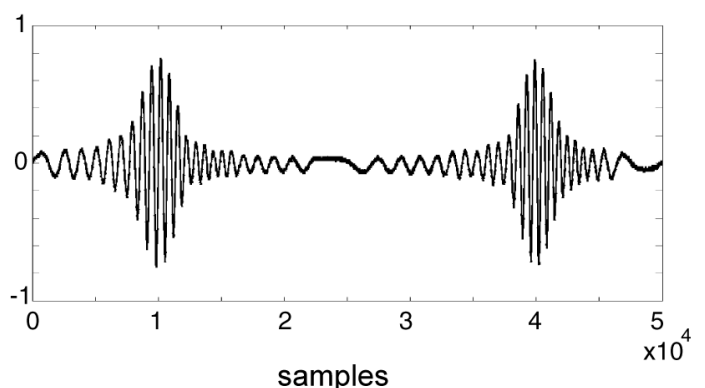

Figure 6. Experimental results of the effect of the edge on the visibility, with a bandwidth of $150 \mathrm{~nm}$ and a central wavelength of $575 \mathrm{~nm}$; going from (a) to (e), the star is moved from the centre of the central step to the centre of the next step. In (a) and (e), because the star is focused in the centre of the step, one fringe set is observed. In (b), as the star is moved close to the edge, a second fringe set appears, with contrast smaller than the other component. In (c), the star is focused approximately in the middle of the edge, both fringe sets present similar contrast. In (d), as the star is moved towards the centre of the next step, the contrast of the first fringe set decreases and the contrast of the second one increases.

nents show similar contrast, and their addition is 0.91 . From Fig. $6 \mathrm{~d}$, the addition of the two components is 0.90 , and finally in Fig. 6e the fringe when the star is focused in the next step is plotted, and the visibility is 0.78.

The difference in contrast from 0.82 to 0.78 between the case when the star is on-axis with respect to the case when the star is off-axis is attributed to dispersion effects. To compare the experimental results with the analytical predictions, we calculate the edge factor using Equations 4 and 5. The average from the three measurements taken when the star is 
focused on the edge is multiplied by the edge factor to obtain the visibility. This resulting visibility is compared with the reference visibility obtained by averaging the two visibilities taken when the stars are focused on the steps. To calculate the edge factor, it is necessary to measure the depth of the steps. From a direct measurement, the depth of the steps of the staircase mirror used in the setup is $2.65 \mu \mathrm{m}$, which agrees quite well with the $2.64 \mu \mathrm{m}$ calculated from the measurement of the peak-to-peak distance of the two fringe sets. The edge factor calculated with the parameters of the experiment is then $1.13 \pm 0.01$. The average of the addition of the two contributions to the visibility is $0.91 \pm 0.02$. From Equation 3 a visibility of $0.80 \pm 0.02$ is recovered, where the error is calculated by error propagation. Given that the average of the visibility measured when the star is focused in the steps is $0.80 \pm 0.02$. This result shows that it is possible to recover the visibility when the star is focused on the edge with similar accuracy as the visibility of a star located in the centre of the step.

\subsection{Experimental results with a $34 \mathrm{~mm}$ baseline}

For the experiment described in this section we changed the baseline of the interferometer to $34 \mathrm{~mm}$. In order to use the same mirror, we also have to change the effective focal length of the setup to $7.438 \mathrm{~m}$ to keep the relation between the depth and the width of the steps, as shown in Eq. 1. When changing the focal length, the positions of the stars in the focal plane change, and this allows to have a reference star in a step and another star in the edge between two steps. The results are depicted in Fig. 7. The visibility of the reference star, plotted in Fig. $7 \mathrm{a}$, is $0.70 \pm 0.02$. It is lower than expected due to dispersion effects. The calibrated fringe from the star focused on the edge between two steps is shown in Fig. 7b. The addition of the two visibility components is equal to $0.80 \pm 0.02$. Because the edge factor depends on the depth of the steps and the bandwidth and we have not change these parameters, it is $1.13 \pm 0.01$ as in the previous case. With this factor, a visibility of $0.71 \pm 0.02$ is recovered, and the relative phase is $(3.0 \pm 0.1) \times 10^{-4} \mathrm{rad}$.
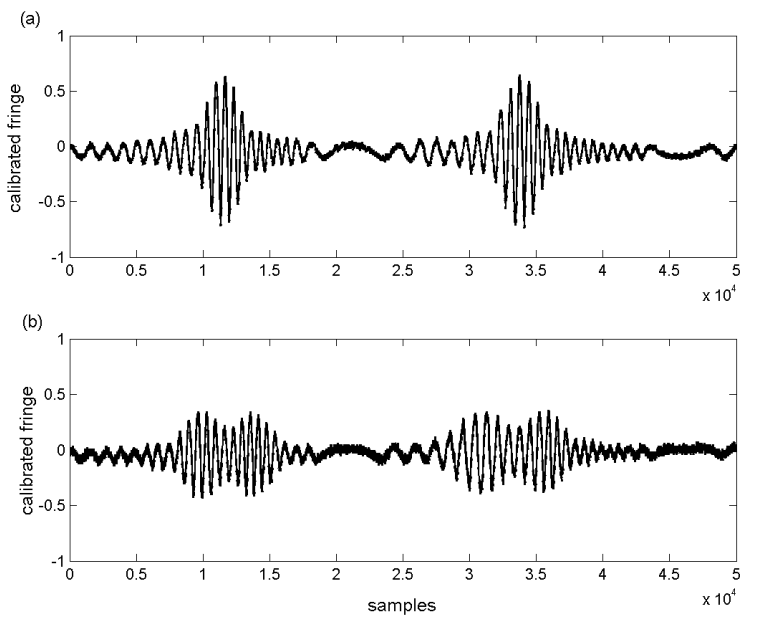

Figure 7. Experimental results of the effect of the edge on the visibility, with a bandwidth of $150 \mathrm{~nm}$, a central wavelength of $575 \mathrm{~nm}$, and a baseline of $34 \mathrm{~mm}$ : (a) the calibrated fringe from the reference star, (b) the calibrated fringe from the star focused on the edge between two steps.

\section{CONCLUSIONS}

We have demonstrated a new approach to the field-of-view problem in optical interferometry. The main advantage of our approach is that a wide field of view is achieved in one shot, it is very flexible and compatible with image or pupil plane combination interferometers. The compatibility with pupil plane interferometers allows beam combination with a signalto-noise ratio larger than the homothetic mapping solution with Fizeau or imaging Michelson-type interferometers. Experimental results have been shown that prove the feasibility of the concept. Fringes from an off-axis star separated 1.07 arcmin from the on-axis reference star have been obtained simultaneously and with a contrast similar to the on-axis one. The relative phase of the off-axis star has been retrieved and corresponds to the expected value. In the case when a star is focused on the edge of a step, the modulated fringe pattern is split into two fringe sets, but the addition of the individual contrasts of the two sets, apart from a proportionality constant, yields the expected visibility as if the star was focused only 
on one step. This constant is the so-called edge factor and can be obtained from the spectrum of the detected light and the mirror configuration. Experimental results show that the visibility is recovered within a $1 \%$ error. Therefore, the discontinuous nature of our wide-field solution does not imply that the acquired field of view is discontinuous. Using the simple algorithm described in this paper, we show that a continuous wide field of view can be acquired in one shot with a Michelson pupil-plane interferometer.

\section{ACKNOWLEDGMENTS}

The authors would like to thank the Knowledge Center for Aperture Synthesis, a collaboration of TNO and Delft University of Technology, for financial support. We also would like to thank Philips Research Laboratories, for manufacturing the staircase mirror. We greatly acknowledge Aad van der Lingen for his very helpful technical support during the assembly and alignment of the setup.

\section{REFERENCES}

1 E. Pedretti, A. Labeyrie, L. Arnold, N. Thureau, O. Lardiere, A. Boccaletti, and P. Riaud, "First images on the sky from a hyper telescope", Astronomy and Astrophysics Supplement Series 147, 285-290 (2000).

2 S. Gillet, P. Riaud, O. Lardiere, J. Dejonghe, J. Schmitt, L. Arnold, A. Boccaletti, D. Horville, and A. Labeyrie, "Imaging capabilities of hypertelescopes with a pair of micro-lens arrays", Astronomy and Astrophysics 400, 393-396 (2003).

3 I. Montilla, S. F. Pereira, and J. J. M. Braat, "Michelson wide-field stellar interferometry: principles and experimental verification", Appl Optics submitted (2004).

$4 \quad$ M. Schoeller, "Fizeau beam combination with optical interferometers: some ideas for second-generation VLTI instrumentation", in Interferometry in Optical Astronomy, P. Lena and A. Quirrenbach eds., Proc. SPIE 4006, 116-123 (2000).

5 A. Labeyrie, "Resolved imaging of extra-solar planets with future 10-100km optical interferometric arrays." Astronomy and Astrophysics Supplement Series 118, 517-524 (1996).

6 I. Montilla, E. J. Bakker, S. F. Pereira, and J. J. M. Braat, "A new concept for wide field imaging", in Interferometry for Optical Astronomy II, W. Traub eds., Proc. SPIE 4838, 416-424 (2003).

7 I. Montilla, J. Sellos, S. F. Pereira, and J. J. M. Braat, "Visibility retrieval in the presence of discontinuous path length compensation", Appl Optics submitted (2004). 